\title{
Effet de la matrice de l'eau sur l'élimination des micropolluants organiques par ozonation. Partie 2. Simulation de l'élimination d'un micropolluant dans les réacteurs idéaux Effect of water composition on organic micropollutant removal by ozonation Parti II : Simulation of micropollutant removal in ideal reactors
}

\author{
M. T. Orta de Velasquez, N. Martin, V. Bodison et A. Laplanche
}

Volume 7, numéro 3, 1994

URI : https://id.erudit.org/iderudit/705203ar

DOI : https://doi.org/10.7202/705203ar

\section{Aller au sommaire du numéro}

\section{Éditeur(s)}

Université du Québec - INRS-Eau, Terre et Environnement (INRS-ETE)

\section{ISSN}

0992-7158 (imprimé)

1718-8598 (numérique)

\section{Découvrir la revue}

Citer cet article

Orta de Velasquez, M. T., Martin, N., Bodison, V. \& Laplanche, A. (1994). Effet de la matrice de l'eau sur l'élimination des micropolluants organiques par ozonation. Partie 2. Simulation de l'élimination d'un micropolluant dans les réacteurs idéaux. Revue des sciences de l'eau / Journal of Water Science, 7(3), 309-323. https://doi.org/10.7202/705203ar

\section{Résumé de l'article}

L'équation cinétique qui permet de calculer l'oxydation d'un micropolluant dans les réacteurs d'ozonation s'écrit:

$-(\mathrm{d}[\mathrm{P}] / \mathrm{dt})=\left(\mathrm{K}_{\mathrm{D}}\left[\mathrm{O}_{3}\right]_{\mathrm{L}}+\mathrm{K}_{\mathrm{ID}}\left[\mathrm{OH}^{\circ}\right]\right)[\mathrm{P}]$

Kd et Kid: constantes de vitesse de l'ozone et des radicaux hydroxyles sur le micropolluant P.

Dans la première partie, l'approche théorique de la concentration en radicaux hydroxyles a montré que $[\mathrm{OH} \cdot]$ est proportionnel à la concentration en ozone $([\mathrm{OH} \cdot]=$ $\left.\mathrm{k}^{\prime}[03]\right)$. On a donc:

$(\mathrm{d}[\mathrm{P}] / \mathrm{dt})=\mathrm{K}_{\mathrm{G}}\left[\mathrm{O}_{3}\right]_{\mathrm{L}[\mathrm{P}]}$ with $\mathrm{K}_{\mathrm{G}}=\mathrm{K}_{\mathrm{D}}+\mathrm{K}_{\mathrm{ID}} \mathrm{K}^{\prime}$

Dans un réacteur parfaitement agité, les concentrations en ozone et en micropolluant sont constantes et l'élimination s'écrit:

$([\mathrm{P}] /[\mathrm{Po}])=\left(1 / 1+\mathrm{K}_{\mathrm{G}}\left[\mathrm{O}_{3}\right]_{\mathrm{L}} \tau\right)$ with $\tau=(\mathrm{V} / \mathrm{Q})$

Dans un réacteur piston, les concentrations varient tout au long de la colonne et il est habituel de modéliser un tel réacteur comme un grand nombre de R.P.A. en série de volume DeltaV et de hauteur DeltaH (Dans notre approche DeltaH = 0,01 m).

Dans les deux cas, la simulation de l'élimination du micropolluant est basée sur la connaissance de la valeur de $\mathrm{kG}$ et de la concentration en ozone dans l'eau [03]L [03]L est obtenue de la résolution des bilans massiques dans un volume $\mathrm{V}$ ou $\sim \mathrm{V}$. ozone à l'entrée + ozone transféré = ozone à la sortie + ozone consommé L'ozone transféré utilise pour son calcul des relations semi-empiriques donnant la constante de Henry et la valeur du kLa.

L'ozone consommé est déduit de la relation établie dans la partie 1: $\left(\mathrm{d}\left[\mathrm{O}_{3} \mathrm{l}_{\mathrm{L}} / \mathrm{dt}\right)=\mathrm{w}\left[\mathrm{O}_{3}\right]_{\mathrm{L}}\right.$

Les résultats de la simulation sont comparés aux résultats expérimentaux obtenus avec un pesticide organo-phosphoré, le parathion. Les paramètres variables sont le temps de contact (300 - 600 s), le $\mathrm{pH}(6,7-8,2)$ et le taux de traitement $\left(1 \mathrm{à} 5 \mathrm{~g} / \mathrm{m}^{3}\right)$. Une valeur de $\mathrm{kG}$ comprise entre 500 et $600 \mathrm{M}^{-1} \mathrm{~s}^{-1}$ donne une bonne corrélation entre les valeurs expérimentales et calculées.

Cependant, on peut noter quelques différences, en particulier dans la partie basse de la colonne, ce qui montre la nécessité de prendre en compte pour des calculs plus précis l'hydrodynamique du réacteur.

L'emploi du programme de simulation permet de tracer deux abaques qui montrent l'influence pour n'importe quel micropolluant des facteurs kGteta et w. 


\title{
Effet de la matrice de l'eau sur l'élimination des micropolluants organiques par ozonation \\ Partie II : Simulation de l'élimination \\ d'un micropolluant dans les réacteurs idéaux
}

\author{
Effect of water composition on organic \\ micropollutant removal by ozonation \\ Part II : Simulation of micropollutant removal in ideal reactors
}

\section{M.T. ORTA DE VELASQUEZ1', N. MARTIN³, V. BOISDON44, A. LAPLANCHE2}

Reçu le 20 septembre 1993 , accepté le 28 juin $1994^{*}$.

\section{SUMMARY}

Micropollutant (P) oxidation in an ideal ozonation reactor uses the kinetic equation :

$-\frac{d[P]}{d t}=\left(k_{D}\left[O_{3} k+k_{I D}\left[O H^{\circ}\right]\right)[P]\right.$

$k_{D}$ et $k_{I D}$ : kinetic rate constant of ozone and hydroxy radicals on the micropollutant $P$.

In part 1, the theoritical equation shows that $\left[\mathrm{OH}^{\circ}\right]$ is proportional to the ozone concentration $\left(\left[\mathrm{OH}^{\circ}\right]=k^{\prime}\left[\mathrm{O}_{3}\right]\right)$ and thus the following equation is obtained :

$-\frac{d[P]}{d t}=k_{G}\left[O_{3}\right]_{L}[P] \quad$ with $k_{G}=k_{D}+k_{I D} k^{\prime}$

In a completely stirred tank reactor, ozone concentration in liquid phase is constant and pesticide elimination is given by the equation :

$$
\frac{[P]}{[P o]}=\frac{1}{1+k_{G}\left[O_{3}\right]_{L} \tau} \quad \text { with } \tau=\frac{V}{Q}
$$

In a plug flow reactor, ozone concentration in liquid phase varies along the column. To modelize them, we use the model of completely stirred tank reactors in series where the unit volume is $\Delta V$. In our calculations, this volume is obtained by $S$ (reactor cross section area) and $\Delta$ h equal to $0,01 \mathrm{~m}$. In this volume $\Delta V$, ozone and micropollutant concentrations are considered as a constant.

1. Instituto de Ingenieria, Ed.5, Coordinación de Ingeniería Ambetial, Universidad Nacional, Autonoma de México, 04511 Coyoacán, México, D.F.

2 Laboratoire Chimie des Nuisances et Génie de I'Environnement, ENSCR, Avenue du Général Leclerc, 35700 Rennes Beaulieu.

3 Trailigaz, Compagnie Générale de l'Ozone, 29-31 Boulevard de la Muette, 95145 Garges-Les-Gonesse.

4 Anjou Recherche, Chemin de la Digue, BP 76, 78603 Maisons-Laffitte.

* Les commentaires seront reçus jusqu'au 31 mars 1995. 
Simulation calculations are based on the knowledge of global kinetic constant $\mathbf{k}_{\mathbf{G}}$ and ozone concentration.

The value of the ozone concentration is obtain from mass balances on the oxidant (on a $\Delta V$ or $V$ volume reactor) :

ozone inlet + transferred ozone $=$ consumed ozone + ozone outlet

The quantities of transferred ozone are calculated from the Henry law and a semi empirical $\mathbf{k}_{\mathbf{L}}$ a equation.

The quantity of consumed ozone is calculated from the equation in part I

$-\frac{d\left[O_{3}\right]_{L}}{d t}=w\left[O_{8}\right]_{L}-$

Experimental results are obtained with parathion, an organo-phosphorus pesticide on a bubble column pilot plant :

Parameters are contact time $(300-600 \mathrm{~s}), \mathrm{pH}(6,7-8,2)$ and ozone treatment rate (1 to $5 \mathrm{~g} / \mathrm{m}^{3}$ ).

$A k_{G}$ value of 500 or $600 \mathrm{M}^{-1} \mathrm{~s}^{-1}$ shows a good correlation between predicted and simulated pesticide concentrations.

However, there are noticable differences, especially at the bottom of the column. This shows the necessity to take into account the hydrodynamic properties of the reactor during next works.

The use of the simulation program lets to calculate the elimination of pesticide versus the two main parameters : the factor $k_{G} \tau$ and the value of $w$.

Key-words : ozone, organic micropollutant, pesticide, bubble tower, simulation.

L'équation cinétique qui permet de calculer l'oxydation d'un micropolluant dans les réacteurs d'ozonation s'écrit :

$-\frac{d[P]}{d t}=\left(k_{p}\left[O_{3} L+k_{I D}\left[O H^{\circ}\right]\right)[P]\right.$

$k_{D}$ et $k_{I D}$ : constantes de vitesse de l'ozone et des radicaux hydroxyles sur le micropolluant $P$.

Dans la première partie, l'approche théorique de la concentration en radicaux hydroxyles a montré que $\left[\mathrm{OH}^{\circ}\right]$ est proportionnel à la concentration en ozone $\left(\left[\mathrm{OH}^{\circ}\right]=\mathrm{k}^{\prime}\left[\mathrm{O}_{3}\right]\right)$. On a donc :

$-\frac{d[P]}{d t}=k_{G}\left[O_{3}\right]_{L}[P] \quad$ avec $k_{G}=k_{D}+k_{I D} k^{\prime}$

Dans un réacteur parfaitement agité, les concentrations en ozone et en micropol luant sont constantes et l'élimination s'écrit :

$\frac{[P]}{[P o]}=\frac{1}{1+k_{G}\left[O_{3}\right]_{L} \tau} \quad$ avec $\tau=\frac{V}{Q}$

Dans un réacteur piston, les concentrations varient tout au long de la colonne et il est habituel de modéliser un tel réacteur comme un grand nombre de R.P.A. en série de volume $\Delta V$ et de hauteur $\Delta h$ (Dans notre approche $\Delta h=0,01 \mathrm{~m}$ ).

Dans les deux cas, la simulation de l'élimination du micropolluant est basée sur la connaissance de la valeur de $\mathrm{k}_{\mathrm{G}}$ et de la concentration en ozone dans l'eau $\left[\mathrm{O}_{3} l_{\mathrm{L}}\right.$, 
$\left[\mathrm{O}_{3}\right]_{\mathrm{L}}$ est obtenue de la résolution des bilans massiques dans un volume $\mathrm{V}$ ou $\Delta \mathrm{V}$. ozone à l'entrée + ozone transféré = ozone à la sortie + ozone consommé

L'ozone transféré utilise pour son calcul des relations semi-empiriques donnant la constante de Henry et la valeur du $\mathbf{k}_{L^{2}}$.

L'ozone consommé est déduit de la relation établie dans la partie 1 :

$-\frac{d\left[O_{3}\right]_{L}}{d t}=w\left[O_{3}\right]_{L}$

Les résultats de la simulation sont comparés aux résultats expérimentaux obtenus avec un pesticide organo-phosphoré, le parathion. Les paramètres variables sont le temps de confact $(300-600 \mathrm{~s})$, le $\mathrm{pH}(6,7-8,2)$ et le taux de traitement $(1$ à $5 \mathrm{~g} / \mathrm{m}^{3}$ ).

Une valeur de $k_{G}$ comprise entre 500 et $600 \mathrm{M}^{-1} \mathrm{~s}^{-1}$ donne une bonne corrélation entre les valeurs expérimentales et calculées.

Cependant, on peut noter quelques différences, en particulier dans la partie basse de la colonne, ce qui montre la nécessité de prendre en compte pour des calculs plus précis l'hydrodynamique du réacteur.

L'emploi du programme de simulation permet de tracer deux abaques qui montrent l'influence pour n'importe quel micropolluant des facteurs $\mathbf{k}_{\mathbf{G}} \tau$ et $\mathbf{w}$.

Mots clés : ozone, micropolluant organique, modélisation, pesticides.

\section{1 - INTRODUCTION}

L'utilisation de l'ozone dans le traitement des eaux d'alimentation répond à un double but : une bonne désinfection de l'eau distribuée et une élimination des composés organiques indésirables, composés toxiques ou générant des nuisances (odeurs et saveur).

De plus, les eaux de consommation distribuées doivent être conformes à un certain nombre de normes: vis-à-vis des micropolluants organiques notamment, l'eau distribuée ne doit pas contenir plus de $0,1 \mu \mathrm{g} / \mathrm{l}$ d'un pesticide donné et plus de $0,5 \mu \mathrm{g} / \mathrm{l}$ de l'ensemble des pesticides. Le taux des hydrocarbures polycycliques aromatiques doit être inférieur à $0,2 \mu \mathrm{g} / \mathrm{l}$ et celui des phénols à $10 \mu \mathrm{g} / \mathrm{l}$. II est donc particulièrement intéressant pour l'exploitant d'une usine de production de prévoir l'abattement d'un micropolluant le long de la chaîne de traitement et en particulier dans l'unité d'oxydation à l'ozone.

En utilisant, pour établir les bilans massiques sur l'ozone, les résultats obtenus dans la $1^{\text {re }}$ partie, nous allons définir les éléments nécessaires à la conception d'un modèle de simulation, comparer les résultats de la modélisation à ceux obtenus sur pilote pour un pesticide, le parathion, et montrer l'influence de quelques paramètres sur la dégradation d'un micropolluant, notamment la valeur du taux spécifique de consommation de l'oxydant $\mathrm{w}$. 


\section{2 - PARAMÈTRES NÉCESSAIRES À LA MOdÉLISATION DE L'ÉLIMINATION D'UN MICROPOLLUANT}

Une colonne d'ozonation à bulles est un réacteur gaz liquide dans lequel l'élimination des micropolluants organiques dépend de la concentration des espèces oxydantes en solution. Pour l'ozone, il y a un transfert gaz liquide avec réactions chimiques à l'intérieur de la phase liquide.

Pour modéliser ce type de réacteurs, il faut connaître les réactions mises en jeu et leur cinétique, les équations de transfert et l'hydrodynamique du réacteur.

\subsection{Cinétique de l'élimination d'un polluant organique}

Dans un réacteur d'ozonation, un polluant organique est éliminé soit par action directe de l'ozone avec une constante de vitesse $k_{\mathrm{D}}$, soit par une action indirecte due à la production de radicaux $\mathrm{OH}^{\circ}$ avec une constante de vitesse $k_{10}$.

En faisant, d'après la bibliographie (LANGLAIS et al., 1991 ; DORE, 1989), l'hypothèse raisonnable que les deux réactions ont des cinétiques globales du $2^{\mathrm{e}}$ ordre, on peut écrire pour un composé organique quelconque :

$$
-\frac{d[P]}{d t}=k_{D}\left[O_{3} h[P]+k_{1 D}\left[\mathrm{OH}^{\circ}\right][P]\right.
$$

Comme nous l'avons montré dans la partie l et également d'après GLAZE et KANG (1989), la production de radicaux $\mathrm{OH}^{\circ}$ est fonction de la concentration en ozone,

$$
\left[\mathrm{OH}^{\circ}\right]=\mathrm{k}^{\prime}\left[\mathrm{O}_{3}\right]_{\mathrm{L}}
$$

$k^{\prime}$ étant une fonction complexe faisant intervenir les caractéristiques de l'eau à traiter ( $\mathrm{pH}$, matières organiques, alcalinité) et la quantité d'eau oxygénée formée ou ajoutée.

Il est donc possible d'écrire:

$$
-\frac{d[P]}{d t}=k_{G}\left[O_{3} h[P] \quad \quad \quad \text { avec } k_{G}=k_{D}+k_{1 D} k^{\prime}\right.
$$

La valeur de $\mathrm{k}_{\mathrm{G}}$ variera selon le micropolluant $\left(\mathrm{k}_{\mathrm{D}}\right.$ et $\left.\mathrm{k}_{\mathrm{ID}}\right)$ et selon la compo. sition de l'eau et la quantité d'initiateurs $\left(\mathrm{H}_{2} \mathrm{O}_{2}\right)$ rajoutés $\left(\mathrm{k}^{\prime}\right)$.

\subsection{Application de la cinétique aux réacteurs idéaux}

En assimilant le réacteur à bulles à un réacteur à une phase liquide dont le volume réel $V=(1-\varepsilon)$ Vo (Vo: volume du réacteur; $\varepsilon$ : pourcentage du volume occupé par la phase gazeuse) et en considérant que l'ozone et les radicaux $\mathrm{OH}^{\circ}$ ont des vitesses de réaction d'ordre deux $\left(r=k_{1 D}\left[\mathrm{OH}^{\circ}\right][\mathrm{P}]\right.$ ou $r=k_{D}\left[O_{3}\right][P]$, LEVENSPIEL (1972) rapporte les équations suivantes : 
- Pour un réacteur parfaitement agité (RPA) dont la caractéristique est une concentration uniforme pour tout le réacteur égale à la concentration de sortie, l'élimination d'un micropoltuant s'écrit :

$$
\frac{[\mathrm{P}]}{[\mathrm{Po}]}=\frac{1}{1+\mathrm{k}_{\mathrm{G}}\left[\mathrm{O}_{3}\right]_{\mathrm{L}} \tau} \quad \text { avec } \tau=\frac{\mathrm{V}}{\mathrm{Q}_{\mathrm{L}}}
$$

- Pour un réacteur piston, les concentrations varient tout au long du cheminement de la phase liquide. Pour effectuer le calcul, nous avons utilisé le concept qu'un réacteur piston est modélisé par un grand nombre de RPA en série. Le réacteur a đonc été divisé en petits éléments de volume $\Delta \mathrm{V}$ et de hauteur $\Delta \mathrm{h}=0,01 \mathrm{~m}$. Pour cháque élément, on considère les concentrations en espèces réactives constantes. Le modèle calcule ainsi l'avancement de la réaction de dégradation du micropolluant le long du réacteur piston.

\subsection{Le transfert de l'ozone}

Sur le petit élément de volume $\Delta V$ du réacteur, en faisant l'hypothèse que la concentration de l'ozone dans la phase gazeuse reste constante, la quantité d'ozone transféré s'écrit :

$$
\mathrm{O}_{3, T}=\mathrm{k}_{\mathrm{L}} \cdot \mathrm{a} \cdot \Delta \mathrm{V}\left(\left[\mathrm{O}_{3}\right]^{\star}-\left[\mathrm{O}_{3}\right]_{\mathrm{L}}\right)
$$

$k_{L} \quad$ étant le coefficient de transfert de film $(\mathrm{m} / \mathrm{s})$

a l'aire d'échange volumique $\left(\mathrm{m}^{2} / \mathrm{m}^{3}\right)$

$\left[\mathrm{O}_{3}\right]^{*}$ la concentration de l'ozone à l'interface $\left(\mathrm{mole} / \mathrm{m}^{3} \mathrm{ou} \mathrm{g} / \mathrm{m}^{3}\right)$

$\left[\mathrm{O}_{3}\right]_{\mathrm{L}}$ la concentration de l'ozone en phase liquide (mole/m $\mathrm{m}^{3}$ ou $\mathrm{g} / \mathrm{m}^{3}$ )

Dans cette équation, on considère que l'ozone étant un gaz peu soluble, toute la résistance au transfert de masse se trouve dans le film liquide. $\left[\mathrm{O}_{3}\right]^{*}$ sera relié à la pression partielle et donc à la concentration de l'ozone en phase gazeuse $\left[\mathrm{O}_{3}\right]_{\mathrm{G}}\left(\mathrm{mole} / \mathrm{m}^{3}\right)$ par la relation de Henry.

Pression partielle d'ozone en phase gazeuse $=\mathrm{He}\left[\mathrm{O}_{3}\right]^{*}$.

La constante de Henry He peut être calculée par de nombreuses relations semi-empiriques qui prennent en compte soit la température, soit la température et le pH (PERRY, 1984 ; OUDERNI et al., 1987 ; ROTH et SULLIVAN, 1981 ; LANGLAIS et al., 1991).

Les calculs présentés utilisent cette dernière relation :

$\log \mathrm{He}=22,3-4030 / \mathrm{T} \quad$ He étant exprimé en atm/fraction molaire

\subsection{Le calcul de $k_{L} a$}

Le $k_{L}$ a est a priori un paramètre qui va dépendre des conditions de l'exploitation (débits, taux de traitement...) mais également des paramètres propres du contacteur (homogénéité, vieillissement des poreux...).

En faisant des mesures de l'ozone dans l'eau et dans l'air, à la sortie d'un contacteur, il est toujours possible d'évaluer expérimentalement une valeur moyenne de $k_{L} a$. 
Dans un programme de simulation, il sera possible:

- soit d'utiliser les formules générales établies par AKITA et YOSHIDA (1974) pour les colonnes à bulles;

- soit d'utiliser, comme le fait notre programme, des relations simplifiées qui ne prennent en compte que la vitesse superficielle du gaz $U_{S G}$. La formulation générale est :

$$
k_{L} a=\alpha U_{S G}^{\beta} \quad k_{L} \text { a en } \min ^{-1} \text { et } U_{S G} \text { en } m \cdot h^{-1}
$$

avec $\alpha=0,06$ et $\beta=0,6$ pour un réacteur pilote (LAPLANCHE et al, 1991)

ou $\alpha=0,013$ et $\beta=0,95$ pour une cuve industrielle à $t=12^{\circ} \mathrm{C}$

$\alpha=0,0135$ et $\beta=0,82$ pour une cuve industrielle à $t=5^{\circ} \mathrm{C}$

(ROUSTAN et al., 1977)

\subsection{Bilans massiques sur l'oxydant}

Avant d'écrire les équations de bilan sur l'air et sur l'eau, il faut rappeler les hypothèses de travail :

- Quel que soit le type d'écoulement de la phase aqueuse, l'air ozoné s'écoule en flux piston.

- Le transfert de l'ozone est en régime permanent à température constante et le $\mathrm{k}_{\mathrm{L}}$ a est pris constant le long de la colonne.

- Pour une unité de volume $\Delta V$ correspondant à une hauteur $\Delta h$, les concentrations en ozone dans l'eau et dans l'air sont homogènes et égales à la concentration de sortie.

- II n'y a pas de gradient de concentration radial dans la colonne: elles sont homogènes dans un plan à une hauteur donnée du réacteur.

- Il n'y a pas d'accélération de transfert de masse due aux réactions chimiques de l'ozone (Nombre de Hatta $<0,3$ ).

- Les équations sont établies pour des flux à contre-courant.

Pour l'air, le bilan massique entre les cotes $h$ et $h+\Delta h$ s'écrit :

$$
Q_{G}\left[O_{3}\right]_{G, h+\Delta h}=Q_{G}\left[O_{3}\right]_{G, h}+k_{L} a \Delta V\left(\left[O_{3}\right]_{h}{ }^{*}-\left[O_{3}\right]_{L, h}\right)
$$

et pour l'eau

$$
\left.Q_{L}\left[O_{3}\right]_{L, h}+\Delta h=Q_{L}\left[O_{3}\right]_{L, h}+k_{L} a \Delta V\left(\left[O_{3}\right]_{h}{ }^{*}-\left[O_{3}\right]_{L, h}\right)-\left[O_{3}\right]_{L, h}\left(w+k_{D}[P]\right)\right) \Delta V
$$

\subsection{Principe du calcul et organigramme}

$\mathrm{Si}$ on appelle la cote 0 le haut du réacteur (entrée de l'eau) et la cote $\mathrm{H}$ le bas du réacteur (entrée de l'air ozoné), le calcul est initié par une valeur arbitraire de $\left[\mathrm{O}_{3}\right]_{\mathrm{G}, 0}$. Après une première boucle, on compare la valeur calculée $\left[\mathrm{O}_{3}\right]_{\mathrm{G}, \mathrm{H}}$ à la valeur réelle et on opère par dichotomie.

L'organigramme du calcul est donné par la figure 1. 


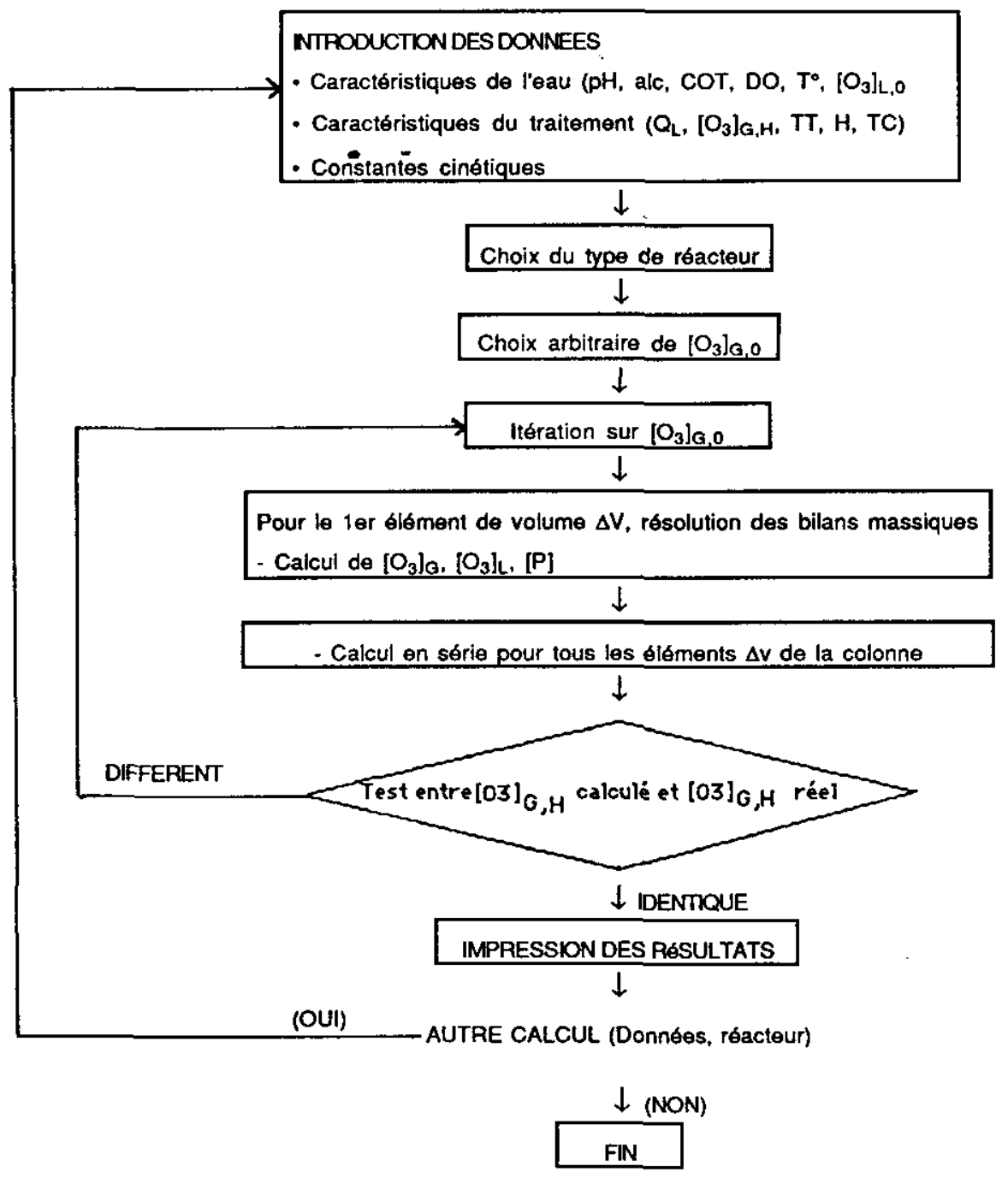

Figure 1 Organigramme général.

General program flowchart. 
Tableau 1 Résultats expérimentaux (LAPLANCHE et al., 1974).

Table 1 Experiment results (LAPLANCHE et al., 1974).

\begin{tabular}{|c|c|c|c|c|c|c|c|c|c|c|c|c|c|c|c|c|c|c|c|c|c|}
\hline \multirow{2}{*}{ Expérience } & \multirow{2}{*}{$\begin{array}{c}\text { T.T. } \\
\text { parathion } \\
\text { résiduel }\end{array}$} & \multicolumn{4}{|c|}{$1 \mathrm{mg} / \mathrm{l}$} & \multicolumn{4}{|c|}{$2 \mathrm{mg} / \mathrm{l}$} & \multicolumn{4}{|c|}{$3 \mathrm{mg} / \mathrm{l}$} & \multicolumn{4}{|c|}{$4 \mathrm{mg} / \mathrm{l}$} & \multicolumn{4}{|c|}{$5 \mathrm{mg} / \mathrm{l}$} \\
\hline & & $G_{1}$ & $\mathrm{G}_{2}$ & $G_{3}$ & $G_{4}$ & $G_{1}$ & $\mathrm{G}_{2}$ & $G_{3}$ & $\mathrm{G}_{4}$ & $G_{1}$ & $\mathrm{G}_{2}$ & $\mathrm{G}_{3}$ & $\mathrm{G}_{4}$ & $G_{1}$ & $\mathrm{G}_{2}$ & $G_{3}$ & $\cdot \mathrm{G}_{4}$ & $G_{1}$ & $\mathrm{G}_{2}$ & $G_{3}$ & $\mathrm{G}_{4}$ \\
\hline 1 & $\%$ & 96 & 90 & 51 & 36 & 92 & 75 & 8 & 6 & 80 & 50 & 3 & 0 & 13 & 20 & 0 & 0 & 12,5 & 8 & 0 & 0 \\
\hline$\|$ & $\%$ & 91 & 78,5 & 21 & 13,5 & 83,5 & 62 & 6 & 0 & 70 & 36,5 & 0 & 0 & 12,5 & 12 & 0 & 0 & 28 & 0 & 0 & 0 \\
\hline III & $\%$ & 98 & 95 & 40 & 30 & 90,5 & 72,5 & 40 & 10 & 77 & 39 & 3 & 0 & 28 & 11,5 & 1 & 0 & 28,5 & 8,5 & 0 & 0 \\
\hline IV & $\%$ & 97 & 80 & 13 & 10 & 83 & 55 & 3,5 & 3,5 & 71 & 13 & 2 & 1 & 3 & 3 & 0 & 0 & 15 & 2,5 & 0 & 0 \\
\hline
\end{tabular}

\begin{tabular}{|c|c|c|c|c|}
\hline Expériences & $\begin{array}{l}1 \\
1 \\
\text { III } \\
N\end{array}$ & $\begin{array}{l}\mathrm{pH}=8,2 ; \\
\mathrm{pH}=8,2 ; \\
\mathrm{pH}=6,7 ; \\
\mathrm{pH}=6,7 ;\end{array}$ & $\begin{array}{l}\mathrm{TC}=360 \mathrm{~s} ; \\
\mathrm{TC}=720 \mathrm{~s} ; \\
\mathrm{TC}=360 \mathrm{~s} ; \\
\mathrm{TC}=720 \mathrm{~s} ;\end{array}$ & $\begin{array}{l}{\left[\mathrm{O}_{3}\right]_{G, 0}=12 \mathrm{gNm^{3 }}} \\
{\left[\mathrm{O}_{3}\right]_{G, 0}=12 \mathrm{gNm} \mathrm{Nm}^{3}} \\
{\left[\mathrm{O}_{3}\right]_{G, 0}=12 \mathrm{gNm^{3 }}} \\
{\left[\mathrm{O}_{3}\right]_{G, 0}=12 \mathrm{~g} \mathrm{Nm}^{3}}\end{array}$ \\
\hline
\end{tabular}




\section{3 - VAlidation du PROGRAMME dE SIMULATION}

Afin de montrer la validité du modèle, nous allons comparer les valeurs calculées avec celles obtenues expérimentalement sur une tour d'ozonation pilote de $4 \mathrm{~m}$ de haut et de $0,15 \mathrm{~m}$ de diamètre.

Le micropolluant dégradé est du parathion dont la constante d'action directe est $\mathrm{k}_{\mathrm{D}}=70 \mathrm{M}^{-1} \mathrm{~s}^{-1}$ (LAPLANCHE et al., 1976). L'eau est dopée avec du parathion avec une concentration $[\mathrm{Po}]=70$ à $80 \mu \mathrm{g} / \mathrm{l}$ et les autres caractéristiques sont $\mathrm{T}=20^{\circ} \mathrm{C}, \mathrm{COT}=3,5 \mathrm{mg} / \mathrm{l}$, absorbance à $254 \mathrm{~nm}=0,05$ et alcalinité $=60 \mathrm{mg} / \mathrm{l}$ (exprimée en $\mathrm{CaCO}_{3}$ ). Les paramètres expérimentaux sont le temps de contact (360 et 720 s), le pH de l'eau $(6,7$ et 8,2 ) et le taux de traitement ( 1 à $5 \mathrm{~g}$ d'ozone $/ \mathrm{m}^{3}$ d'eau).

Le parathion résiduel a été mesuré au quart (point $\mathrm{G}_{1}$ ), à la moitié (point $G_{2}$ ), au trois quart (point $G_{3}$ ) et à la sortie de l'eau de la colonne (point $G_{4}$ ).

L'ensemble des résultats expérimentaux est donné dans le tableau 1 (d'après LAPLANCHE et al., 1974).

Sur cette colonne pilote, le kLa a été déterminé expérimentalement par LAPLANCHE et al. (1991):

$$
k_{L} a=0,06 U_{S G}^{0,61}
$$

Au niveau du comportement hydraulique, ce réacteur réel ne correspond pas directement à un réacteur piston. En effectuant des traçages, LE SAUZE et al. (1992) ont montré que J, nombre de RPA en série modélisant ce réacteur, est compris entre 6 et 15 selon les conditions de débit d'air et d'eau.

Tableau 2 Valeurs de J en sortie de la colonne (LE SAUZE et al., 1992).

Table 2 Experimental values of $\mathrm{J}$.

\begin{tabular}{|lllll|}
\hline \multicolumn{1}{|c}{ Qair $\mathrm{V} / \mathrm{h}$} & 212 & 150 & 72 & 0 \\
\hline $1397(\mathrm{TC}=193 \mathrm{~s})$ & $\mathrm{J}=14$ & $\mathrm{~J}=14$ & $\mathrm{~J}=15$ & $\mathrm{~J}=27$ \\
$904(\mathrm{TC}=299 \mathrm{~s})$ & $\mathrm{J}=13$ & $\mathrm{~J}=14$ & $\mathrm{~J}=15$ & $\mathrm{~J}=17$ \\
$327(\mathrm{TC}=588 \mathrm{~s})$ & $\mathrm{J}=6$ & $\mathrm{~J}=7$ & $\mathrm{~J}=8$ & $\mathrm{~J}=21$ \\
\hline
\end{tabular}

\subsection{Approche de la valeur de la constante $\mathbf{k}_{\mathrm{G}}$}

Nous avons testé notre modèle de simulation avec plusieurs valeurs arbitraires de la constante globale $k_{G}$. Pour les valeurs de 300, 500 et 1000 $\mathrm{M}^{-1} \mathrm{~s}^{-1}$, la comparaison entre courbes calculées et simulées est donnée par les figures 2 et 3 . 

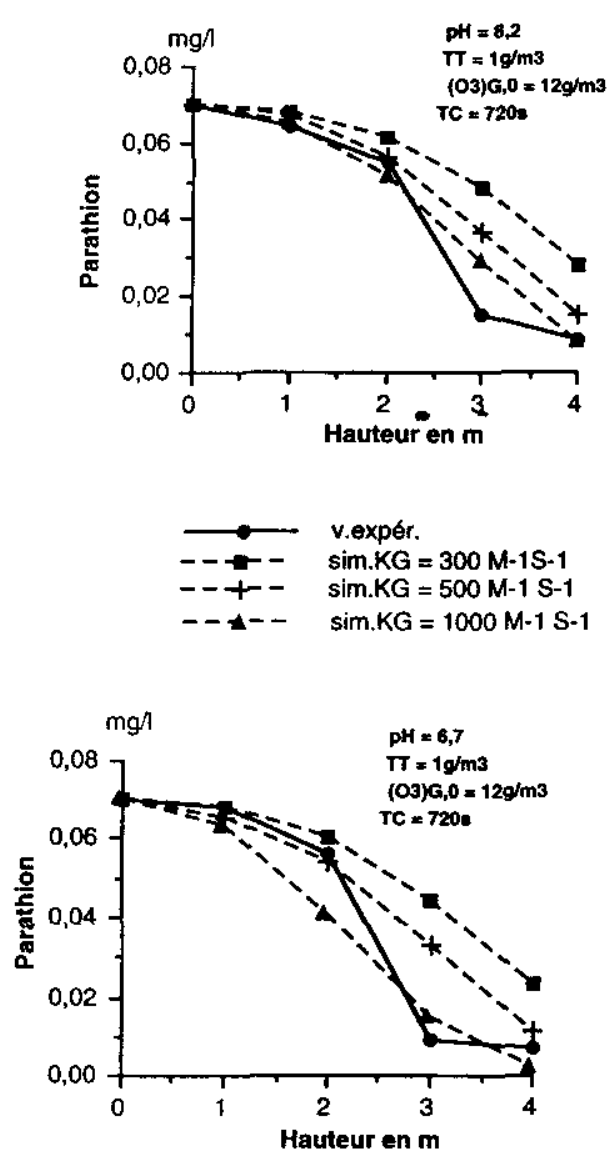
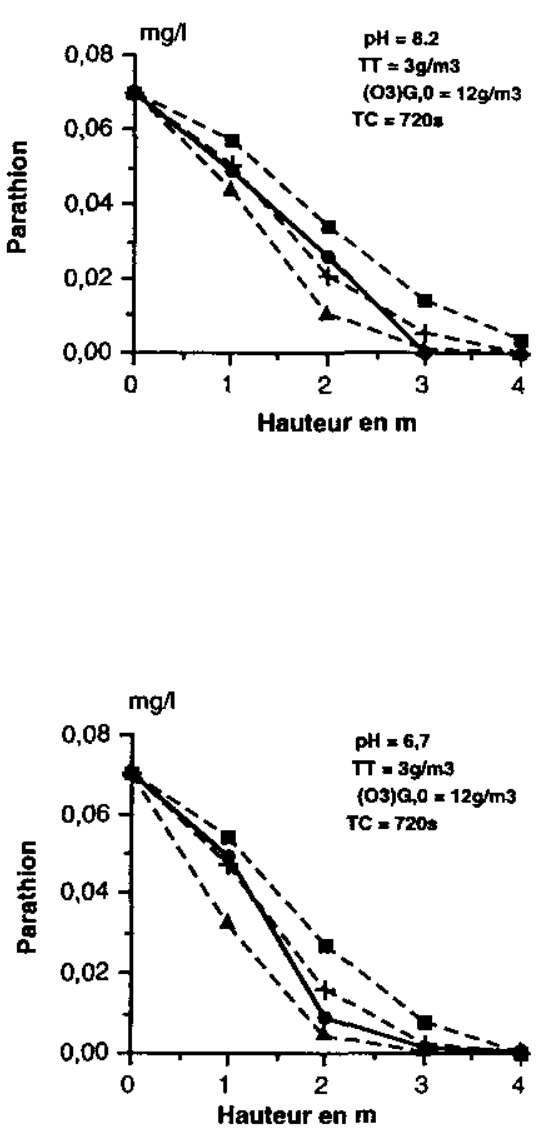

Figure 2 Influence du $\mathrm{k}_{\mathrm{G}}$ sur l'élimination du parathion.

Comparaison entre les valeurs simulées et les valeurs mesurées.

Parathion removal; influence of global kinetic rate constant $k_{G}$. Comparison between experimental et simulated data.

La visualisation de ces résultats permet de donner les conclusions suivantes :

- La valeur de la constante $k_{G}$ est évidemment primordiale pour simuler la dégradation du pesticide. Dans le cas du parathion et de l'eau de Rennes, on obtient une assez bonne corrélation pour $\mathrm{k}_{\mathrm{G}} \# 500 \mathrm{M}^{-1} \mathrm{~s}^{-1}$.

- L'adéquation est correcte pour les points $G_{1}, G_{2}$ et $G_{4}$ (ce dernier constitue la valeur la plus importante à retenir) mais la valeur expérimentale du point $\mathrm{G}_{3}$ s'écarte beaucoup de la courbe calculée. Ce phénomène est imputable au fait que la dispersion dans le réacteur n'est pas homogène. La zone basse du réacteur qui correspond à l'arrivée de l'air ozoné a un comportement proche d'un réacteur parfaitement agité (LE SAUZE et al., 1992). Ceci explique le décrochage de la courbe expérimentale au niveau du point $\mathrm{G}_{3}$ et la quasi égalité des valeurs pour $\mathrm{G}_{3}$ et $\mathrm{G}_{4}$. Pour une simulation beaucoup plus fine, il faudra donc tenir compte de l'hydrodynamique du réacteur. 

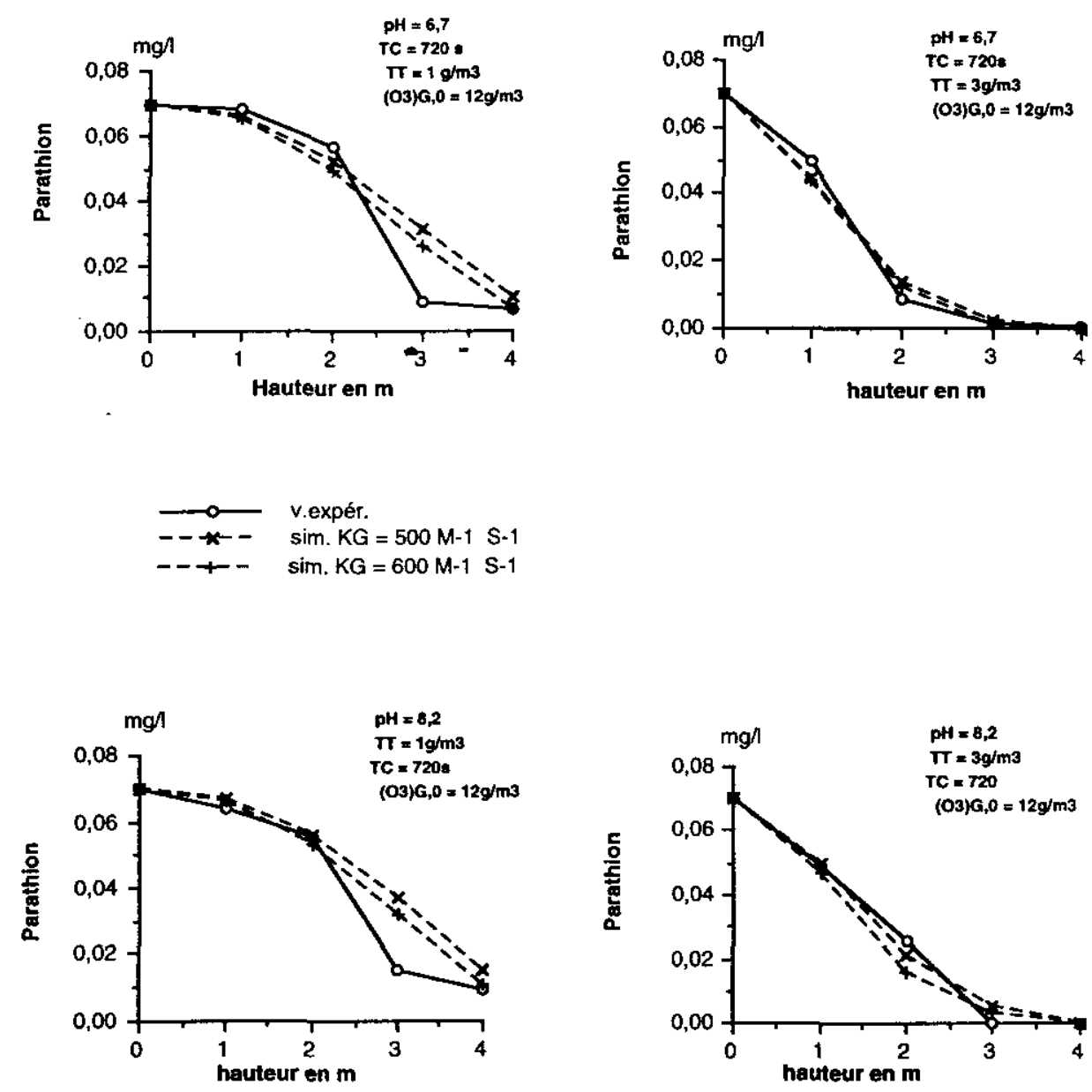

Figure 3 Elimination du parathion; influence de l'hydrodynamique du réacteur. Comparaison entre les valeurs expérimentales et calculées.

Parathion removal; influence of the hydrodynamic of the reactor. Comparison between experimental et simulated data.

- Les valeurs respectives de $k_{D}$ et $k_{G}$ étant 70 et $\approx 500 \mathrm{M}^{-1} \mathrm{~s}^{-1}$, il est aisé de conclure que l'élimination du parathion est essentiellement due au mécanisme radicalaire. Ceci sera vrai pour la grande majorité des pesticides pour lesquels les constantes $k_{D}$ sont toujours faibles (HAAG et YAO, 1992).

La méthode ne permet pas d'accéder à une valeur précise de $\mathrm{k}_{\mathrm{G}}$ car des simulations effectuées avec les valeurs 500 ou $600 \mathrm{M}^{-1} \mathrm{~s}^{-1}$ ne permettent pas de trancher (fig. 3). Toutefois, ces courbes montrent la bonne concordance entre les valeurs mesurées et calculées quels que soient le $\mathrm{pH}$ et le taux de traitement. Au niveau de l'influence de la réaction radicalaire, il sera dont judicieux de travailler avec les vraies valeurs de $k_{I D}$ et une relation empirique de calcul de $k$ ' sur laquelle portent nos travaux actuels. 


\subsection{Influence des différents paramètres}

L'utilisation du programme de simulation permet de voir l'influence de quelques paramètres de l'ozonation. Nous avons retenu $w$ qui influe sur les concentrations en ozone dissous, $\mathrm{k}_{\mathrm{G}}$ qui représente les réactions chimiques et $k_{\mathrm{G}} \tau$, nombre de Damköhler qui montre l'influence du couple « réaction-temps de contact " pour une réaction de pseudo $1^{\mathrm{er}}$ ordre $\left(\left[\mathrm{O}_{3}\right]_{\mathrm{L}}=\right.$ constante $)$.

La figure 4 est un abaque montrant la dégradation d'un micropolluant le long d'une colonne en fonction de $k_{G}$ dans des conditions moyennes standards $\left(T C=360 \mathrm{~s} ; T \mathrm{~T}=1 \mathrm{~g} / \mathrm{m}^{3} ;\left[\mathrm{O}_{3}\right]_{\mathrm{G}, 0}=18 \mathrm{~g} / \mathrm{m}^{3} ; \mathrm{k}_{\mathrm{L}} \mathrm{a}=0,16.10^{-2} \mathrm{~s}^{-1}\right.$; $\mathrm{pH}=7,5$; COT $=2 \mathrm{mg} / \mathrm{l}$; Absorbance à $254 \mathrm{~nm}=0,12$; Alcalinité $=$ $150 \mathrm{mg} / \mathrm{l}$ en $\mathrm{CaCO}_{3}$ et $\mathrm{T}^{\circ}=20^{\circ} \mathrm{C}$ ).

La figure 5 est un abaque donnant l'influence de $w$ en fonction du couple $\mathrm{k}_{\mathrm{G}} \mathrm{t}$ pour un taux de traitement de $1 \mathrm{~g} / \mathrm{m}^{3}$.

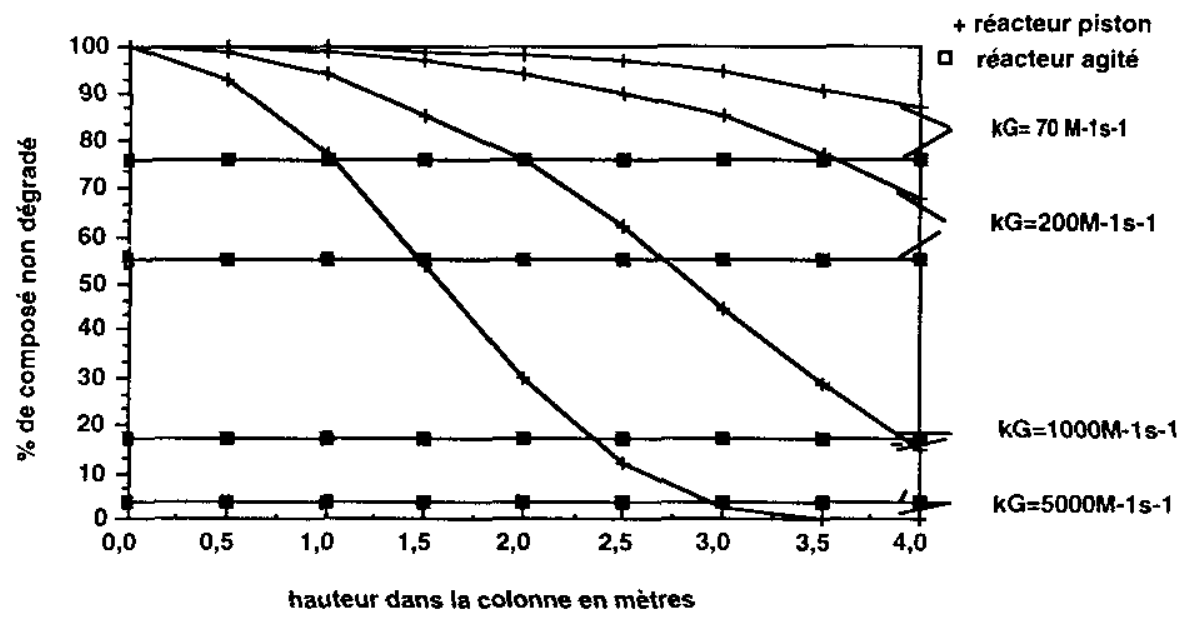

Figure 4 Elimination d'un micropolluant. Influence de la constante cinétique globale $\mathrm{k}_{\mathrm{G}}$ et comparaison des réacteurs idéaux.

Micropolluant removal along an ideal rector. Influence of the global kinetic rate constant $k_{G}$.

\section{a) Influence de la constante cinétique globale $k_{G}$}

Si on regarde les valeurs de $\mathrm{k}_{\mathrm{G}}\left(\mathrm{M}^{-1} \mathrm{~s}^{-1}\right)$, on peut noter qu'une dégrada tion convenable du micropolluant ne sera obtenue que pour $\mathrm{k}_{\mathrm{G}}>1000$ et une dégradation quasi totale pour $\mathrm{k}_{\mathrm{G}} \approx 5000$. Cela veut dire que pour beaucoup de micropolluants tels que les pesticides $\left(k_{D}\right.$ du parathion $=70 \mathrm{M}^{-1} \mathrm{~s}^{-1}$, LAPLANCHE et al., $1976 ; k_{D}$ de l'atrazine $=13 \mathrm{M}^{-1} \mathrm{~s}^{-1}$, YAO et HAAG, 1992), l'élimination se fera presque exclusivement par un mécanisme radicalaire. Dans ce cas, la valeur de $k_{1 D} k$ ' devra donc être, elle aussi, au moins de l'ordre de $10^{3}$. Pour $k_{1 D}=3.10^{9}$ (valeur moyenne pour un grand nombre de micropolluants), la valeur correspondante de $k^{\prime}$ devra donc être de l'ordre de $3,3.10^{-7}$ et les concentrations respectives en $\mathrm{OH}^{\circ}$ seront $3,3.10^{-12} \mathrm{~mol} / \mathrm{lsi}\left[\mathrm{O}_{3}\right]=10^{-}$ $5 \mathrm{~mol} / \mathrm{l}$ ou $\left[\mathrm{OH}^{\circ}\right]=1,65 \cdot 10^{-12} \mathrm{~mol} / \mathrm{l} \mathrm{si}\left[\mathrm{O}_{3}\right]=0,5 \cdot 10^{-5} \mathrm{~mol} / \mathrm{l}$. 


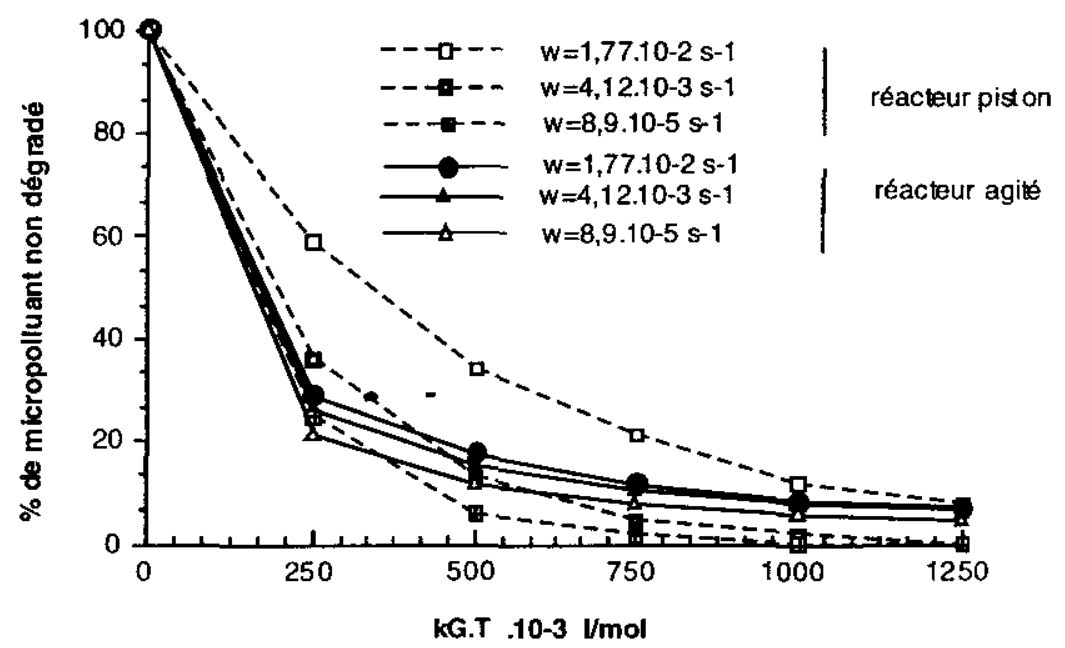

Figure 5 Elimination d'un micropolluant. Influence du nombre de Damköhler $\mathrm{k}_{\mathrm{G}} \tau$ et du taux spécifique de consommation de l'ozone $w$.

Micropolluant removal along an ideal rector. Influence of Damköhler number $k_{G} \tau$ et du taux spécifique de consommation de l'ozone $w$.

En régime permanent, l'équation de bilan sur l'oxydant est satisfaite. Pour un RPA, l'équilibre est atteint sur la totalité du réacteur alors que pour un RP. l'équilibre est réalisé pour chaque petit élément de volume $\Delta \mathrm{V}$. Cela correspond à une courbe d'ozone dissous d'allure exponentielle dont l'aire totale sous la courbe est inférieure à celle observée dans le cas du RPA. Globalement, cela modifie les règles classiques de comparaison des RPA et RP car la quantité totale d'oxydant mise en jeu est plus importante dans le RPA que dans le piston. Par exemple, dans les conditions simulées (Figure 4), le point d'isorendement est obtenu pour un taux de transformation de $82 \%$. Pour des taux de transformation plus faibles, le RPA est plus performant alors que pour des taux plus importants, on revient à la prépondérance du RP.

\section{b) Influence du produit $k_{G} \tau$ et de $w$}

Il est encore plus intéressant pour chaque taux de traitement de voir l'influence du nombre de Damköhler, $\mathrm{k}_{\mathrm{G}} \tau$ en $1 . \mathrm{mol}^{-1}$, couplée à la qualité de l'eau à traiter qui est représentée par la valeur de $w$.

La figure 5 visualise les résultats pour un taux de traitement de $1 \mathrm{~g} / \mathrm{m}^{3}$ et pour trois valeurs de $w$ qui couvrent la gamme des points expérimentaux de la partie 1.

Sans parler de la production des radicaux libres qui jouera à la fois sur la valeur de $k_{G}$ par l'intermédiaire de $k^{\prime}$ et sur la valeur de $\left[\mathrm{O}_{3}\right]$, il est possible de faire les commentaires suivants :

- L'effet de w sur l'élimination du micropolluant n'aura un rôle significatif que lorsque I'hydrodynamique du réacteur se rapprochera d'un flux piston et que le taux de transformation sera inférieur à $80 \%$. 
- Avec un réacteur parfaitement agité, il sera très difficile d'éliminer plus de 90 à $95 \%$ du composé organique. Ceci veut dire que pour un pesticide qui entre a une concentration supérieure à $2 \mu \mathrm{g} /$ litre dans le réacteur d'ozonation, si celui-ci est assimilable à un RPA, la concentration de sortie dépassera la norme limite imposée par la réglementation européenne $(0,1 \mu \mathrm{g} / \mathrm{litre})$.

- Enfin, il faudra éviter les conclusions hâtives en ce qui concerne l'élimination d'un micropolluant pour un taux de traitement donné lors du couplage $\mathrm{H}_{2} \mathrm{O}_{2} / \mathrm{O}_{3}$, car la plupart du temps, le fait d'augmenter la teneur en radicaux et donc la valeur de $k^{\prime}$ aura pour conséquence une diminution rapide de la concentration en ozone. Le résultat final sera donc une conjugaison de ces deux phénomènes.

\section{CONCLUSION}

Cette première approche basée sur les réacteurs idéaux montre qu'il est possible de simuler l'élimination d'un micropolluant sur les tours d'ozonation à bulles. Les expériences effectuées sur le parathion prouvent que l'utilisation d'un tel programme permet ensuite de calculer directement l'effet du changement d'un paramètre (dose d'oxydant, temps de contact, qualité de l'eau...). Pour être plus performant, il faudra également prendre en compte de nouveaux paramètres qui ont été approximés dans cette approche.

En premier lieu, il faut établir une relation empirique qui permette de relier la concentration en radicaux libres $\left[\mathrm{OH}^{\circ}\right]$ aux différentes concentrations en oxydants $\left(\mathrm{O}_{3}, \mathrm{H}_{2} \mathrm{O}_{2}\right)$ et à la qualité de l'eau. Une telle relation, même approchée, permettra de simuler le comportement de n'importe quel micropolluant à condition de connaître les constantes $k_{D}$ et $k_{I D}$.

Dans un autre domaine, il est important de mieux cerner le comportement hydrodynamique du réacteur. En se basant sur les courbes de distribution de temps de séjour, LE SAUZE et al. (1992) ont montré qu'il était possible pour une installation pilote de distinguer 3 zones (arrivée de l'eau, arrivée de l'air et partie médiane), chacune étant caractérisée par J RPA en série. Sur des cuves industrielles, ROUSTAN et al. (1993) propose une simulation comportant 2 types d'écoulement en série, l'un étant piston pour les zones non ozonées, l'autre modélisé par J RPA en série pour les zones ozonées. Nous envisageons la possibilité d'introduire ces différents modèles hydrauliques dans le programme présenté.

Enfin, chaque unité d'ozonation sera caractérisée par une relation donnant un $k_{L}$ a moyen qui lui sera propre. Si le programme de simulation peut proposer des formules empiriques de calcul du $k_{L} a$, pour simuler le transfert d'une manière fine, il faudra laisser à l'utilisateur la possibilité d'introduire ses propres données. 


\section{RÉFÉRENCES BIBLIOGRAPHIQUES}

AKITA K., YOSHIDA F., 1974. "Bubble size, interfacial area and liquid mass transfer coefficients in bubble columns". Indus. Eng. Chem. Proc. Dev, 13, (1), 84.

DORE M., 1989. "Chimie des oxydants et traitement des eaux ". Technique \& Documentation, Lavoisier (PARIS).

GLAZE W.H., KANG J.W., 1989. "Evaluation of the ozone. Hydrogen peroxide process using tetrachloroethylene as a model compound ". 9th Ozone World Congress, New-York, 1, 596.

LANGLAIS B., RECKOW D., BRINK D., 1991. «Ozone in water treatment. Application and Engineering ". Lewis Publishers.

LAPLANCHE A., MARTIN G., RICHARD Y., 1974. "Ozonation d'une eau polluée par du parathion ". TSM L'eau, 7, 407.

LAPLANCHE A., MARTIN G., RICHARD Y., 1976. "Etude de la cinétique de f'ozonation des pesticides organo-phosphorés Exemple du Parathion". TSM L'eau, 4, 169.

LAPLANCHE A., LE SAUZE N., MARTIN G., LANGLAIS B., 1991. "Simulation of ozone transfer in water. Comparison with a pilot unit ". Ozone Sci. Eng., 13 (5) 535.

LE SAUZE N., LAPLANCHE A., MARTIN N., MARTIN G., 1993. « Modelling of ozone transfer in a bubble column *. Wat. Res., 27, (6), 1071.
LE SAUZE N., LAPLANCHE A., ORTA DE VELASQUEZ M.T., MARTIN N., LANGLAIS B., MARTIN G., 1992. "The residense time distribution of the liquid phase in a bubble column and its effect on the ozone transfer ". Ozone Sci. Eng, 14, (3),245.

LEVENSPIEL 0., 1972. "Chemical reaction engineering ". John Wiley and sons, New York.

OUDERN! A., MORA J., BES R., 1987. "Ozone absorption in water, mass transfer and solubility". Ozone Sci. Eng. ,9, (4), 391.

PERRY F., 1984. "Chemical Engineering Handbook ». 6ème Edition, Mac Gnaw Hill.

ROTH J., SULLIVAN D., 1981. "Solubility of ozone in water". Indus. Eng. Chem. Fund., 20, 137.

ROUSTAN M., LINE A., DUGUET J.P., MALLEVIALLE J., WABLE U., 1993. "Modelling Hydraulics of ozone contactors ". Ozone Sci. Eng., 15, (3), 213.

ROUSTAN M., DUGUET J.P., MALEVIALLE J., 1977. "Mass balance analysis of ozone in conventionnal bubble contactors". Ozone Sci. Eng, 9, (3), 289.

YAO C.C.D., HAAG W.R., 1982. "Rate constant for reaction of hydroxyl radicals with several drinking water contaminants ". Envir. Sci. Technol, 26, (5), 1005. 